\title{
Nonlinear RF spurious in a cylindrical cavity with superconducting endplates
}

\author{
Jordi Mateu, Carlos Collado, Timothy J. Shaw, Juan M. O’Callaghan * \\ Universitat Politècnica de Catalunya (UPC), Campus Nord UPC-D3, Jordi Girona 1, Barcelona 08034, Spain
}

\begin{abstract}
We have developed a method to calculate the distribution of fundamental and spurious fields in a metallic cylindrical cavity with superconducting endplates in which signals at two different frequencies are injected. The nonlinearity in the superconductor produces the typical intermodulation effects if the frequencies of the injected signals are sufficiently close to each other and near a resonant mode. Our method uses harmonic balance to match the fields in the cavity with the currents on the endplates. The method can be used for a variety of nonlinear models of the superconducting endplate, and could be the base for a nondestructive procedure to extract the nonlinear parameters of an HTS sample from $\mathrm{RF}$ measurements. Our analysis is restricted to the $\mathrm{TE}_{011}$ mode, but the method can be applied to any propagating mode in the cylindrical cavity. Closed-form equations for the case of square-law nonlinearities in the superconductor are derived and used to check the validity of the harmonic balance calculation.
\end{abstract}

(C) 2002 Elsevier Science B.V. All rights reserved.

Keywords: Cavity resonators; Intermodulation distortion; Nonlinear distortion; Superconducting devices

\section{Introduction}

Most of the work on RF nonlinearities of superconductors has been done on planar resonators and filters. Cavity resonators are also used in filters and in devices for characterization of material properties. In both applications, the nonlinear performance of cavity resonators is of interest. This paper presents a general method to calculate the distribution of spurious fields in a metallic cylindrical cavity with superconducting endplates in the

\footnotetext{
*Corresponding author. Fax: +34-93-401-7232.

E-mail address: joano@tsc.upc.es (J.M. O'Callaghan).

URL: http://www-tsc.upc.es/eef/.
}

$\mathrm{TE}_{011}$ mode. However, with minor modifications, the method is generally applicable to any propagating mode in the cylindrical cavity, to cavities with other cross-sections, or to cavities with a single superconducting endplate.

\section{Nonlinearities in the superconducting endplates}

When the signal levels are small, the electric field on superconducting endplates depends on the surface current density and the surface impedance: $E_{\mathrm{L}}=Z_{\mathrm{s}} J_{\mathrm{s}}$. However, if the currents are large enough, the electric field has a nonlinear dependence with the surface current. Using the nomenclature in [1] we can write the following time-domain equation: 
$e_{\mathrm{NL}}=a_{\mathrm{NL}}\left(j_{\mathrm{s}}\right) j_{\mathrm{s}}+\frac{\mathrm{d}}{\mathrm{d} t}\left[b_{\mathrm{NL}}\left(j_{\mathrm{s}}\right) j_{\mathrm{s}}\right]$,

where $e_{\mathrm{NL}}$ is the additional electric field caused by surface current $j_{\mathrm{s}}$ and by the nonlinearities in the superconductor. Eq. (1) is consistent with the definition of nonlinear surface impedance given in [2]:

$a_{\mathrm{NL}}\left(j_{\mathrm{s}}\right)=\Delta R_{\mathrm{s}}\left(\omega_{0}, j_{\mathrm{s}}\right) ; \quad b_{\mathrm{NL}}\left(j_{\mathrm{s}}\right)=\frac{1}{\omega_{0}} \Delta X_{\mathrm{s}}\left(\omega_{0}, j_{\mathrm{s}}\right)$

and thus $b_{\mathrm{NL}}$ accounts for the departure of the surface reactance from its small-signal values and, as discussed in [1], can be related to a currentdependent penetration depth $\lambda(j)=\lambda(0, T)(1+$ $f(j))$, with $f(0)=0$. This results in $b_{\mathrm{NL}}(j)=$ $\mu_{0} \lambda(0, T) f(j)$.

\section{Field analysis with square-law nonlinearities}

We assume a cylindrical cavity of radius $R$ and height $h$ which is fed with two sinusoidal signals at frequencies $\left(\omega_{1}\right.$ and $\left.\omega_{2}\right)$ which are very close to the resonant frequency of the $\mathrm{TE}_{011}$ mode. If both signals are in resonance, the fields in the cavity are:

$H_{z}=H_{\omega_{i}} J_{0}\left(k_{\rho} \rho\right) \sin (\beta z)$,

$H_{\rho}=-\frac{\beta}{k_{\rho}} H_{\omega_{i}} J_{1}\left(k_{\rho} \rho\right) \cos (\beta z)$,

$E_{\phi}=-j \frac{k \eta}{k_{\rho}} H_{\omega_{i}} J_{1}\left(k_{\rho} \rho\right) \sin (\beta z)$

with $\omega_{i}=\omega_{1}$ or $\omega_{2}, k_{\rho}=3.8317 / R, \beta=\pi / h$. The current density in the superconducting endplates can be calculated from the tangential magnetic field a $z=0$ and $z=h$, so that $J_{\mathrm{s}}=H_{\rho}$. From this, and using $a_{\mathrm{NL}}\left(j_{\mathrm{s}}\right)=\Delta r_{2} j_{\mathrm{s}}^{2}$ and $b_{\mathrm{NL}}\left(j_{\mathrm{s}}\right)=\Delta x_{2} j_{\mathrm{s}}^{2}$ one can calculate the amplitude of the third order intermodulation spurious following the procedure outlined in [3], resulting in a peak field amplitude (i.e., equivalent to $H_{\omega_{i}}$ in Eqs. (3a)-(3c)):

$$
\begin{aligned}
H_{2 \omega_{1}-\omega_{2}}= & 2.3696 \frac{\beta^{4}}{k_{\rho}^{2} \omega_{0} \varepsilon k^{2} \eta^{2} \pi h} Q_{\mathrm{L}} H_{\omega_{1}}^{2} H_{\omega_{2}}^{*} \\
& \times\left[\Delta r_{2}+j \omega_{0} \Delta x_{2}\right] .
\end{aligned}
$$

In the case of a two-port resonator it is straightforward to calculate the power of the intermodulation spurious dissipated at the output port of the resonator from Eq. (4) [3].

\section{Equivalent circuit: general analysis with harmonic balance}

A numerical calculation of the spurious signals generated by the cavity is also possible using harmonic balance [4]. This calculation is not restricted to square-law nonlinearities in the superconducting endplates, and uses the equivalent circuit of the cavity resonator shown in Fig. 1. In this equivalent circuit, the coupling to the cavity is modelled with transformers. As is usually done in the analysis of microwave cavities and waveguides, we have modelled the cylindrical waveguide as a transmission line [5]. The characteristic impedance and complex propagation constant of the line are set to be equal to those of the $\mathrm{TE}_{011}$ mode in the waveguide. With these conditions, the currents and voltages in the line are proportional to the transverse magnetic and electric field in the waveguide. As done in [5], we have set the proportionality constants to make the complex power flow in the

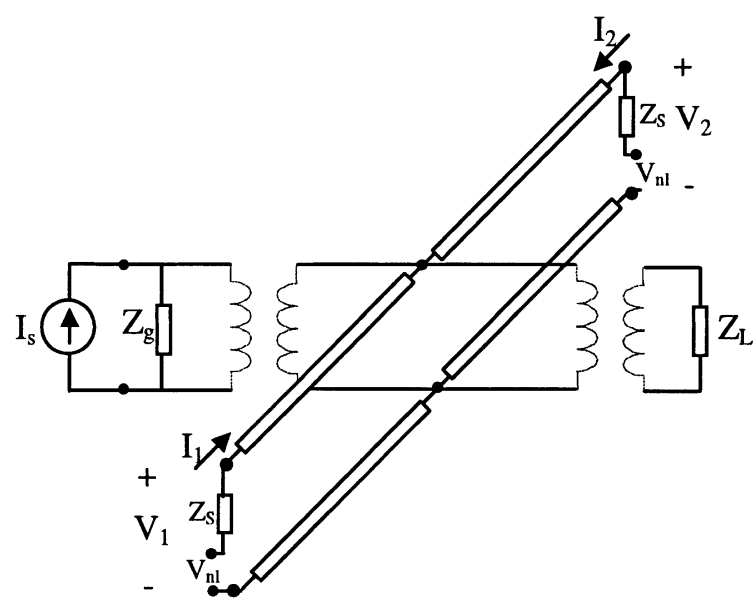

Fig. 1. Equivalent circuit of a two-port cavity resonator. Input and output couplings are modelled with impedance transformers. The superconducting endplates are drawn with a linear impedance $\left(Z_{\mathrm{s}}\right)$ in series with a nonlinear two port to account for the nonlinear effects in the superconductor. 
waveguide equal to that of the transmission line, so that the equivalent circuit can also be used to calculate the power delivered to the output port, and the power available from the source.

With this equivalent circuit, the linear part of the problem is reduced to solving a system of equations in the frequency domain relating the voltage and current in the source to the voltage (transverse electric field) and current (transverse magnetic field) in the upper and lower endplates:

$$
\left[\begin{array}{l}
V_{1} \\
V_{2} \\
V_{\mathrm{s}}
\end{array}\right]=\left[\begin{array}{lll}
Z_{11} & Z_{12} & Z_{13} \\
Z_{21} & Z_{22} & Z_{23} \\
Z_{31} & Z_{32} & Z_{33}
\end{array}\right]\left[\begin{array}{c}
I_{1} \\
I_{2} \\
I_{\mathrm{s}}
\end{array}\right],
$$

where $V_{1}, V_{2}, I_{1}, I_{2}$ represent the equivalent voltages and currents at the upper and lower endplates and $V_{\mathrm{s}}, I_{\mathrm{s}}$ the signal produced by the source. Note that if the source produces sinusoidal signals at several frequencies within the resonant band of the $\mathrm{TE}_{011}$ mode, Eq. (5) has to be replicated for each of the frequencies involved.

In a linear problem, the transverse electric and magnetic fields on the endplates are related through the surface impedance and two additional equations can be added to the system in Eq. (5): $V_{1} / I_{1}=Z_{\mathrm{s}}$ and $V_{2} / I_{2}=Z_{\mathrm{s}}$. These five equations allow the determination of all the variables as a function of the source current $I_{\mathrm{s}}$. This is not possible in the nonlinear case, because the boundary conditions at the endplates are nonlinear and the contribution of the fields generated by the nonlinearities (Eq. (1)) has to be taken into account. To do so, we use an iterative procedure (harmonic balance [4]) very similar to the one described in [6], which starts with the linear solution in frequency domain, transforms the currents in the endplates to time domain, and finds the electric fields caused by the nonlinearity (Eq. (1)). These fields are then transformed back to frequency domain and only the spectral components that fall within the resonant band of the $\mathrm{TE}_{011}$ mode are used for further processing. The equivalent voltages and currents of these spectral components have to match the linear problem described by Eq. (5) at all frequencies of interest (i.e. those of the source currents, and those of the intermodulation products). The iterative procedure is very similar to the one described in [6] and consists in iterating the equiv-

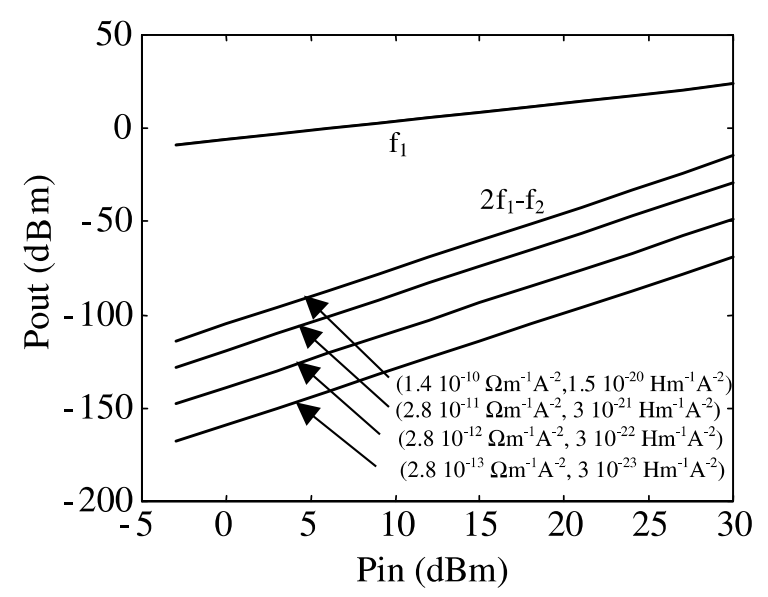

Fig. 2. Output vs. input power for the fundamental and third order intermodulation signals. Square-law nonlinearities are assumed and each trace corresponds to a different $\left(\Delta r_{2}, \Delta x_{2}\right)$ pair. Harmonic balance results agree with analytical simulations to within $<1 \%$.

alent currents on the endplates $\left(I_{1}, I_{2}\right)$ at all the frequencies of interest until the equivalent voltages and currents arising from the linear problem (Eq. (5)) match those imposed by the nonlinearities in the endplates (Eq. (2)).

\section{Results}

Fig. 2 shows an example of the output vs. input power assuming square-law nonlinearities in the superconducting endplates, for various values of $\Delta r_{2}, \Delta x_{2}$. The agreement between the closed-form equations and the harmonic balance results is typically a fraction of a percent. Other outputs of the software include: frequency dependence of the spurious signals, spatial distributions of the spurious, and dependence of the spurious power output with the coupling coefficients. The software has also been run with nonlinearities other than square-law, like the ones suggested in $[2,7]$.

\section{Conclusions}

The RF nonlinear performance of metal cavities with one or two superconducting endplates can be analyzed using harmonic balance. The case of a 
$\mathrm{TE}_{011}$ cylindrical cavity with two superconducting endplates having square-law nonlinearities has been studied, and the analytical results match the results of the numerical simulations. The algorithm developed could be easily adapted to other modes in the cylindrical cavity, to cavities with other cross-sections, or to cavities with a single superconducting endplate.

\section{Acknowledgements}

This work is supported by the Spanish Ministry of Education and Culture through a post-doctoral scholarship for T.J. Shaw (ref. SB9954854667), a pre-doctoral scholarship of J. Mateu (Ref.
AP9978085980), and research grant MAT99-0984C03-03.

\section{References}

[1] J. Mateu, C. Collado, J.M. O'Callaghan, IEEE Trans. Appl. Supercond. 11 (1) (2001) 135.

[2] D.E Oates, A.C. Anderson, S.M. Ali, IEEE Trans. Microw. Theory Tech. 39 (1991) 1522.

[3] T. Dahm, D.J. Scalapino, B.A. Willemsen, J. Appl. Phys. 86 (1999) 4055.

[4] S.A. Maas, Nonlinear Microwave Circuits, Artech House, 1988.

[5] D.M. Pozar, Microwave Engineering, John Wiley \& Sons, Inc., New York, 1998.

[6] C. Collado et al., J. Supercond. 14 (1) (2001) 61.

[7] T. Dahm, D.J. Scalapino, Phys. Rev. B 60 (1999) 13125. 